\title{
Phase-dependent effects in bichromatic high-order harmonic generation
}

\author{
C. Figueira de Morisson Faria, ${ }^{1}$ D. B. Milošević, ${ }^{2, *}$ and G. G. Paulus ${ }^{3}$ \\ ${ }^{1}$ Max Planck Institut für Physik komplexer Systeme, Nöthnitzer Strasse 38, 01187 Dresden, Germany \\ ${ }^{2}$ Max-Born-Institut für nichtlineare Optik und Kurzzeitsspektroskopie, Max-Born-Strasse 2A, 12489 Berlin, Germany \\ ${ }^{3}$ Max Planck Institut für Quantenoptik, Hans-Kopfermann Strasse 1, 85748 Garching, Germany
}

(Received 4 November 1999; published 17 May 2000)

\begin{abstract}
We address high-order harmonic generation with linearly polarized bichromatic fields, concentrating on a modulation in the harmonic yield as a function of the relative phase between the two field components, and on an offset phase shift of this modulation for neighboring cutoff harmonics. These effects have been recently observed in experiments where the relative phase between the two driving fields was controlled. Using the three-step model and the fully numerical solution of the time-dependent Schrödinger equation, we discuss the phase-dependent modulation and show that the offset phase is inherent to a particular set of semiclassical trajectories for the returning electron. These trajectories are identified using classical arguments and isolated by means of the saddle-point method, which allows a detailed investigation of their interference. Thus, by adding a second driving field whose amplitude lies within an adequate parameter range, one is able to single out a set of trajectories according to its behavior with respect to the relative phase. This effect is already present at the the single-atom-response level.

PACS number(s): 42.50.Hz, 32.80.Rm, 32.80.Qk, 42.65.Ky
\end{abstract}

\section{INTRODUCTION}

The emission spectrum of a gaseous sample exposed to a strong laser field covers a long frequency range with harmonics of roughly the same intensities, the so-called "plateau," followed by an abrupt decrease in the harmonic yield, the so-called "cutoff" [1]. A very intuitive and successful description of these features is given by the so-called "threestep model" [2-5]: an electron leaves the atom through tunneling or multiphoton ionization, propagates in the continuum and, depending on its emission time, may be driven back by the field and recombine with its parent ion, such that harmonic radiation up to the extreme ultraviolet (XUV) regime is emitted. Within this picture, the cutoff corresponds to the maximum kinetic energy of the electron upon return and is given, for monochromatic fields, by $\Omega_{\max }=\left|\varepsilon_{0}\right|$ $+3.17 U_{p}$, where $\left|\varepsilon_{0}\right|$ and $U_{p}$ are the ionization potential and the ponderomotive energy, respectively. This model describes existing experiments in some respect even quantitatively and has also been successfully tested against other theoretical methods, such as the fully numerical solution of the time-dependent Schrödinger equation [6], with strikingly similar spectral and temporal profiles, for monochromatic [7-10], bichromatic [11,12], and short-pulsed laser fields [13]. One of the strongest experimental evidences that the physical picture of semiclassical electron trajectories is correct has been provided recently in Ref. [14]. Therein, the trajectories in question have been isolated using effects related to the propagation of the harmonic radiation in the gaseous medium. These experiments have also shown that one can in principle manipulate the harmonic spectra by exploiting particular characteristics of harmonics correspond-

\footnotetext{
* On leave from the Faculty of Science and Mathematics, Department of Physics, University of Sarajevo, Zmaja od Bosne 35, 71000 Sarajevo, Bosnia and Herzegovina.
}

ing to specific trajectories, for instance their different sensitivity to propagation and phase matching.

For a similar reason, i.e., the possibility of coherent control of XUV emission, high-order harmonic generation with bichromatic fields has attracted a lot of attention in the past few years, both theoretically $[11,12,15-18]$ and experimentally [19-21]. In fact, by changing the shape of the bichromatic field, one can in principle manipulate the electron motion in the continuum, suppressing or enhancing particular groups of harmonics. Already for the simplest case, namely a linearly polarized laser field, one can strongly influence the harmonic spectra by varying the field amplitudes, and, in the case of commensurate frequencies, the relative phase $\phi$ between the driving fields. In previous publications, we have shown that the introduction of a second driving field may result in several maximal- and minimal-energy trajectories for the returning electron, which correspond to cutoffs in the emission spectra [11,12], such that the plateau presents a much more complex structure in the bichromatic than in the monochromatic case.

Several parameters determine the prominence of a cutoff in the spectrum: the total field strength at the electron emission time $t_{0}$, the excursion time of the electron in the continuum, and the interference between different semiclassical trajectories. The first parameter is of extreme importance, since the electron leaves the atom with a probability related to the quasistatic ionization rate [22], $\exp \left[-C /\left|E\left(t_{0}\right)\right|\right]$. A very effective way to control the field strength $\left|E\left(t_{0}\right)\right|$ is changing the relative phase between the laser field components of different frequencies. For instance, in [11] we provide an example for which the harmonic yield decreases considerably for a chosen interval of the relative phase where this parameter was particularly weak. Also the interference between the semiclassical trajectories depends very strongly on the relative phase. In principle, slight changes in $\phi$ may radically alter this interference pattern, so that variations of 
orders of magnitude in the harmonic yield are observed, for isolated groups of harmonics [12].

Experimentally, this phase control has been achieved recently for high-order harmonic generation in helium and argon, using a linearly polarized $\omega-2 \omega$ field of comparable intensities [21]. The intensity of the low-frequency field was kept fixed, whereas the high-frequency field and the relative phase $\phi$ were varied. The wavelength of the low-frequency field was approximately $\lambda=800 \mathrm{~nm}$ and intensities of the order of $10^{15} \mathrm{~W} / \mathrm{cm}^{2}$ have been used. In this experiment, the harmonic yield as a function of the relative phase $\phi$, with all other parameters kept constant, exhibits the following main features: (i) The intensity of the cutoff harmonics is modulated. A shift of $2 \pi$ in the relative phase between the $\omega$ and $2 \omega$ fields corresponds to two periods of the modulation of each harmonic. (ii) The modulation itself shows an offset phase shift as the harmonic order is varied: if the modulation of the $n$th harmonic presents a maximum as a function of $\phi$ for a certain phase $\phi_{0}$, then this maximum will be slightly shifted, i.e., it will be at $\phi_{0}+\delta \phi$ for the $(n+1)$ th harmonic. These effects are present within a relatively broad range of intensity ratios $I_{2 \omega} / I_{\omega}$ [21]. The theoretical modeling of these experiments reproduces these findings reasonably well, however, without providing a physical explanation for either the modulation or its phase shift. In [21], as a first approximation, the cutoff energy was taken as the monochromatic value $\Omega_{\max }=\left|\varepsilon_{0}\right|+3.17 U_{p}$ and the ponderomotive energy was related to the low-frequency field only, such that $U_{p}$ was chosen as $U_{p \omega}=I_{\omega} / 4 \omega^{2}$.

In this paper we give a simple explanation of these effects based on the analysis of classical trajectories, within the three-step model and single-atom-response framework. In particular, we isolate the relevant semiclassical trajectories using the saddle-point method, computing the harmonic spectra as the interference between these trajectories. Besides the experimental facts, the conclusions drawn from the classical and semiclassical computations are checked against the results from a fully numerical solution of the time-dependent Schrödinger equation for a one-dimensional model atom with a single bound state, whose energy corresponds to the argon ionization potential. We use atomic units throughout. The paper is outlined as follows: in Sec. II we present our theoretical methods, namely the classical or "simple-man" model (Sec. II A), the saddle-point method (Sec. II B), and the time-dependent Schrödinger equation (Sec. II C). In Sec. III we present and discuss our results and in Sec. IV we state our conclusions.

\section{THEORY}

\section{A. Classical model}

In order to determine the emission and return times and the kinetic energy of the returning electron, we take the numerical solution of the classical equations of motion of an electron in the field

$$
\vec{E}(t)=\hat{\vec{e}}_{x}\left[E_{01} \sin (\omega t)+E_{02} \sin (2 \omega t+\phi)\right],
$$

where $\phi$ denotes the relative phase and $E_{0 i}$ the amplitude of each driving wave. Since all the ensuing motion takes place along the polarization axis, the problem can be treated one dimensionally. At the initial time $t_{0}$, the electron leaves the atom with zero velocity, propagates in the continuum under the influence of the laser field only, and returns to the site of its release at a later time $t_{1}$, such that $x\left(t_{1}\right)=0$. During the process, canonical momentum is conserved. Therefore, the kinetic energy of the electron upon return is given by

$$
E_{\mathrm{kin}}\left(t_{1}, t_{0}\right)=\frac{1}{2}\left[A\left(t_{1}\right)-A\left(t_{0}\right)\right]^{2},
$$

with $A(t)$ being the vector potential, related to the external field by $E(t)=-d A(t) / d t$. This yields a harmonic energy

$$
\Omega_{\mathrm{H}}=\left|\varepsilon_{0}\right|+E_{\text {kin }}\left(t_{1}, t_{0}\right)
$$

by the recombination to the ground state. Following this simple picture, the kinetic energy $E_{\text {kin }}\left(t_{0}, t_{1}\right)$, the emission time $t_{0}$, and the return time $t_{1}$ can be associated to a classical trajectory for the returning electron. The cutoff frequencies are determined by the condition that the kinetic energy is extremal upon return, namely $d E_{\text {kin }}\left(t_{1}, t_{0}\right) / d t_{1}=0$. The emission and return times are connected by the revisiting condition. We use this model either for a single electron, varying the emission time $t_{0}$ within a cycle $T=2 \pi / \omega$ of the low-frequency driving field and calculating $E_{\text {kin }}\left(t_{1}, t_{0}\right)$, which is subsequently plotted as a function of the emission and return times, or we consider an ensemble of electrons whose emission time is varied randomly from 0 to $T$. In this latter case, we select electrons that satisfy the condition $x\left(t_{1}\right)=0$ within a particular set of harmonic energies, given by Eq. (3), and we look at electron counts as functions of the relative phase $\phi$. The contribution of each single electron is weighed with the quasistatic ionization rate $[22,23]$

$$
\Gamma \sim\left[\frac{2^{5 / 2}\left|\varepsilon_{0}\right|^{3 / 2}}{\left|E\left(t_{0}\right)\right|}\right]^{\sqrt{2 / \varepsilon_{0} \mid-1}} \exp \left[\frac{-2^{5 / 2}\left|\varepsilon_{0}\right|^{3 / 2}}{3\left|E\left(t_{0}\right)\right|}\right] .
$$

The first and the second procedures have been used in $[11,12]$ and [24], respectively.

\section{B. Strong-field approximation and saddle-point method}

The classical model discussed in Sec. II A provides useful information concerning the cutoff law and the emission and return times for the electron. However, it does not account for the quantum interference between two or more possible trajectories for the returning electron, which lead to wellstructured harmonic spectra [25-27]. For this purpose, we use a closely related quantum-mechanical approach: the strong-field approximation (SFA) theory of high-order harmonic generation $[3,5,18]$.

Within the SFA, the $n$th harmonic strength is defined as the Fourier component of the time-dependent dipole $[3,5,18]$

$$
\begin{aligned}
D_{n}= & -i \int_{0}^{T} \frac{d t}{T} e^{i n \omega t} \int_{0}^{\infty} d \tau\left(\frac{2 \pi}{i \tau}\right)^{3 / 2} F\left(p_{s} ; t, \tau\right) \\
& \times \exp \left[-i S\left(p_{s} ; t, \tau\right)\right],
\end{aligned}
$$




$$
F\left(p_{s} ; t, \tau\right)=\left\langle\psi_{0}|x| p_{s}-A(t)\right\rangle\left\langle p_{s}-A(t-\tau)|x E(t-\tau)| \psi_{0}\right\rangle,
$$

where $\tau=t-t_{0}$ is the excursion time of the electron in the continuum, $\left|\psi_{0}\right\rangle$ is the atomic ground state, and $p_{s}$ $=\int_{t-\tau}^{\tau} d t^{\prime} A\left(t^{\prime}\right) / \tau$ is the stationary momentum, for which the quasiclassical action

$$
S(p ; t, \tau)=\int_{t-\tau}^{t} d t^{\prime}\left\{\frac{1}{2}\left[p-A\left(t^{\prime}\right)\right]^{2}+\left|\varepsilon_{0}\right|\right\}
$$

satisfies the condition $\nabla_{p} S(p ; t, \tau)=0$. The harmonic yield is proportional to $n^{4}\left|D_{n}\right|^{2}$. The double integral in Eq. (5) can be solved using the saddle-point method (SPM), with the result $[25,26]$

$$
D_{n} \propto \sum_{s} \tau_{s}^{-3 / 2}\left[\operatorname{det}\left(\partial^{2} S_{s}\right)\right]^{-1 / 2} F_{s} \exp \left[i\left(n \omega t_{s}-S_{s}\right)\right],
$$

where $\operatorname{det}\left(\partial^{2} S_{s}\right)$ denotes the determinant of the $2 \times 2$ matrix formed by the second derivatives of the action with respect to $t_{0}$ and $t$ at $p=p_{s}$. For hydrogenlike atoms the product of the dipole matrix element $F_{s}$ can be approximated by ( $n \omega$ $\left.-\left|\varepsilon_{0}\right|\right)^{1 / 2} / n^{3}$. The sum in Eq. (7) extends over all relevant saddle points which satisfy the conditions [18]

$$
\begin{gathered}
\frac{1}{2}\left[p_{s}-A\left(t_{0}\right)\right]^{2}=-\left|\varepsilon_{0}\right|, \\
\frac{1}{2}\left[p_{s}-A(t)\right]^{2}-\frac{1}{2}\left[p_{s}-A\left(t_{0}\right)\right]^{2}=n \omega .
\end{gathered}
$$

We will show in Sec. III that a good approximation for $D_{n}$ can be obtained taking into account only four complex solutions for pairs $\left(t, t_{0}\right)$.

\section{Time-dependent Schrödinger equation}

As our point of reference, we take the one-dimensional time-dependent Schrödinger equation (TDSE) [6] for a single electron subject to a binding potential and a bichromatic field (1). A one-dimensional (1D) model is not so demanding as a full three-dimensional computation and still describes results for linearly polarized fields adequately in qualitative terms [28]. In the velocity gauge, this equation reads

$$
i \frac{d}{d t}|\psi(t)\rangle=\left[\frac{p^{2}}{2}+V(x)-p A(t)\right]|\psi(t)\rangle .
$$

The quadratic term in $A(t)$ was removed by a unitary transformation. The binding potential was chosen

$$
V(x)=-1.1 \exp \left(-x^{2} / 1.21\right),
$$

such that the model atom has a single field-free bound state with energy $\varepsilon_{0}=-0.58$ a.u. According to the experimental conditions the lower frequency of the driving field is taken as $\omega=0.057$ a.u. The power spectra are computed from the time-dependent dipole acceleration $\ddot{x}(t)=\langle\psi(t)|$ $-d V(x) / d x+E(t)|\psi(t)\rangle[29]$.

\section{RESULTS}

\section{A. Upper and lower cutoff branches}

For a monochromatic field, the maximum of $E_{\mathrm{kin}}\left(t_{1}, t_{0}\right)$ is at the well-known value $3.17 U_{p}$, and the semiclassical trajectories originating the cutoff obey a $T / 2$ periodicity. For bichromatic fields, however, this property is maintained only if the higher frequency is an odd multiple of the lower frequency. This is clear, since, for these types of fields, $A(t$ $+T / 2)= \pm A(t)$. On the other hand, if the ratio between the higher and the lower frequency is even, this symmetry is broken. Since the kinetic energy of the electron depends on $A(t)$ according to Eq. (2), this results in a splitting of the cutoff energy into an upper and a lower branch named, respectively, $\Omega_{u}$ and $\Omega_{l}$. This feature can be seen in detail as function of the field strength ratio $E_{02} / E_{01}=\eta$, for $\phi=0$, in a previous publication [12]. These branches behave in strikingly different ways with respect to the relative phase $\phi$. The energy of the upper cutoff branch practically does not vary with this parameter, whereas the lower branch is strongly phase dependent. These features are present for intensity ratios smaller than or of the order of $I_{2 \omega-\omega}=I_{2 \omega} / I_{\omega} \simeq 0.2$. For higher intensity ratios there is a much more complicated pattern for the cutoff energies as functions of the phase. For instance, the case of equally strong driving waves has been discussed in $[11,12]$.

An example is shown in Fig. 1, where $E_{\text {kin }}\left(t_{1}, t_{0}\right)$ is plotted as a function of the emission and return times, for 0 $\leqslant \phi \leqslant 0.2 \pi$ and $\eta=0.32$. This corresponds to an intensity ratio $I_{2 \omega-\omega}=I_{2 \omega} / I_{\omega}=0.1$. Each point $\left(t_{0}, t_{1}, E_{\mathrm{kin}}\left(t_{0}, t_{1}\right)\right)$ in the curves shown fulfills the revisiting condition $x\left(t_{1}\right)=0$, thus characterizing a trajectory for the returning electron. Given an emission time $t_{0}$ on the curve in the lower part of the figure, the return time $t_{1}$ in the corresponding curve in the upper part of the figure is determined by the intersection of the latter curve with a perpendicular line starting from the lower curve at $t_{0}$. The return energy can be read from the ordinate of the graph. The local energy maxima give the cutoffs, and each of these maximal-energy trajectories splits into two, corresponding to a shorter and a longer excursion time for the electron in the continuum. Thus, for a given $E_{\text {kin }}\left(t_{1}, t_{0}\right)$, there may be many possible trajectories for the returning electron. Quantum mechanically, the probability amplitudes related to the electron following each of these trajectories interfere. The cutoff branches $\Omega_{u}$ and $\Omega_{l}$ are marked with thick solid arrows. For this field-strength ratio, the energy of the upper cutoff branch is at roughly $\Omega_{u}$ $=3.8 U_{p}$, whereas $\Omega_{l}$ varies from $2.6 U_{p}$ to $2.3 U_{p}$ for the phase interval in question. In the figure and in the results that follow, the kinetic energy is displayed in units of the ponderomotive energy calculated for the whole field, given by $U_{p}=\Sigma_{n} U_{p n \omega}=E_{01}^{2} / 4 \omega^{2}+E_{02}^{2} / 16 \omega^{2}$, which clearly varies with the intensity ratio. The ponderomotive energy considered in [21], $U_{p \omega}=E_{01}^{2} / 4 \omega^{2}$, is slightly smaller in this case. Roughly, the corresponding emission and return times for the 


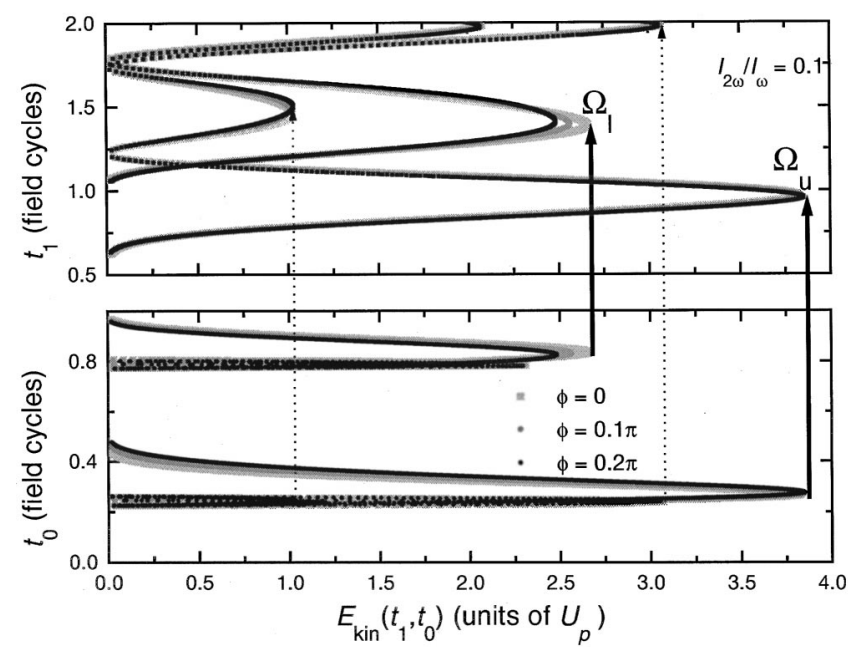

FIG. 1. Classical emission and return times for an electron in a bichromatic field given by Eq. (1) as functions of its kinetic energy upon return, $E_{\mathrm{kin}}\left(t_{1}, t_{0}\right)$, for various relative phases $0 \leqslant \phi \leqslant 0.2 \pi$. The vertical axes in the upper and lower parts correspond, respectively, to the emission and return times, given by $t_{0}$ and $t_{1}$. The field strengths are chosen such that $E_{02} / E_{01}=0.32$, the kinetic energy is given in units of the ponderomotive energy, and the emission and return times in units of the period of the low-frequency field, $T=2 \pi / \omega$. The cutoff energies are marked with arrows connecting both parts of the figure. The thick solid arrows corresponds to the upper and lower cutoff branches and the remaining cutoff energies are indicated by dashed arrows. The influence of the binding potential was neglected.

lower and upper branches are given by, respectively, $\left(t_{1 l}, t_{0 l}\right) \simeq(0.85 T, 1.4 T)$ and $\left(t_{1 u}, t_{0 u}\right) \simeq(0.35 T, 0.9 T)$. Their precise values depend on the relative phase $\phi$. For the parameters considered, the remaining cutoffs, marked with dashed arrows, do not play an important role in the present problem, either for being too near the ionization threshold or due to very long excursion times for the electron, which results in a pronounced wave packet spreading.

A more detailed description of the process above can be obtained using the complex time formalism [25,26]. SPM equations (8), for $\left|\varepsilon_{0}\right| \neq 0$, have only complex solutions $t_{0}$ and $t_{1} \equiv t$. These solutions, for $0 \leqslant t_{0} \leqslant T$ and $0 \leqslant t_{1}-t_{0} \leqslant T$, are presented in Fig. 2. On the left-hand side we present the imaginary part of $t_{0}$ (scaled to the optical cycle $T$ ) as a function of the real part of $t_{0}$, and, similarly, on the righthand side we present solutions for $t_{1}$. The numbers on the curves correspond to the harmonic order $n$ for which solutions were found, for the same intensity ratio as in the previous figure and $E_{01}=0.1$ a.u. It is evident that the solutions $S_{1}$ and $S_{2}$ correspond to the lower cutoff branch, while the solutions $S_{3}$ and $S_{4}$ correspond to the upper one. The cutoffs appear for values of $n$ which correspond to the closest points of the curves $S_{1}$ and $S_{2}$ (or $S_{3}$ and $S_{4}$ ). The physical meaning of the imaginary parts of times $t_{0}$ and $t_{1}$ is connected with the probability that the process in question occurs. This follows from Eq. (7) because, for one particular trajectory, the logarithm of the harmonic yield is proportional to $\operatorname{Im}\left[n \omega t_{1}-S\left(p_{\mathrm{s}}, t_{1}, \tau\right)\right]$. Beyond the points denoted by $n$ $=50$ for the solutions $S_{1}$ and $S_{2}$ (and also by $n=65$ for $S_{3}$
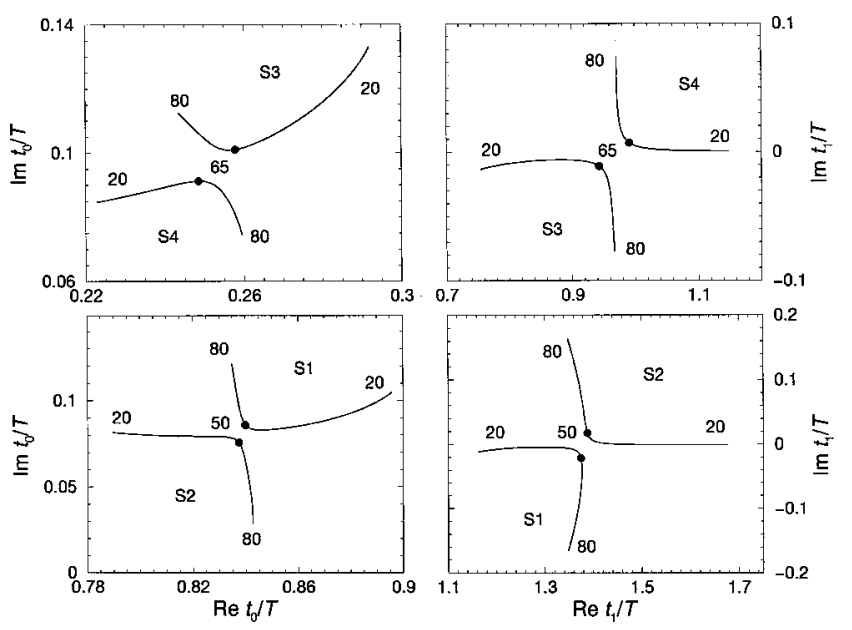

FIG. 2. Complex solutions $t_{0}$ and $t_{1}$ of the SPM equations (8) for a hydrogenlike atomic potential with $\left|\varepsilon_{0}\right|=0.58$ a.u., the same laser field parameters as in Fig. 1, and the relative phase $\phi=0$. Four solutions (denoted by $S_{1}, S_{2}, S_{3}$, and $S_{4}$ ) are obtained for the harmonic order $20 \leqslant n \leqslant 80$. On each curve the beginning (20), the cutoff point (50 or 65), and the end (80) are denoted with the corresponding value of $n$.

and $S_{4}$ ) the imaginary parts increase in absolute value. If these imaginary parts are negative, Eq. (7) gives low emission rates, and, consequently, the position of the cutoff. Otherwise, they lead to an exponential increase in the harmonic yield. More precisely, in the application of Eq. (7) beyond the denoted cutoff points, one of the solutions $S_{1}$ and $S_{2}\left(S_{3}\right.$ and $S_{4}$ ) should be discarded as unphysical. Therefore, for $n>65$ only one trajectory contributes to the harmonic spectra. For $50 \leqslant n \leqslant 65$ three trajectories contribute, while for $n$ $<50$ all trajectories contribute to the harmonic yield.

\section{B. Harmonic spectra}

The importance of each set of trajectories in the harmonic spectra can be inferred from the quantum-mechanical computation. The lower branch is considerably more prominent in the spectra, whereas the most energetic cutoff appears only as a small shoulder. Thus, the "cutoff" seen experimentally corresponds to the strongly phase-dependent set of trajectories. Figures 3 and 4 present some of these spectra, for field strengths $E_{01}=0.1$ a.u. and $E_{02}=0.032$ a.u., which are within the experimental parameter range. For these parameters and $0 \leqslant \phi \leqslant 0.2 \pi$, the upper and lower branches of $\Omega_{\max }$ correspond to the harmonic frequencies $\Omega_{u}=63 \omega$ and $43.1 \omega<\Omega_{l}<47.4 \omega$, respectively. Both cutoff branches are indicated in the figure.

Figures 3(a) and 3(b) present results obtained solving the TDSE (see Sec. II C) and the SFA (see Sec. II B), respectively. Apart from a very good agreement between both results for lower and upper cutoff branches, one observes an energy displacement for the lower branch as $\phi$ is varied, whereas the energy of the upper cutoff branch remains practically inaltered. These results are in perfect agreement with the predictions of Sec. III A obtained using the classical model. 

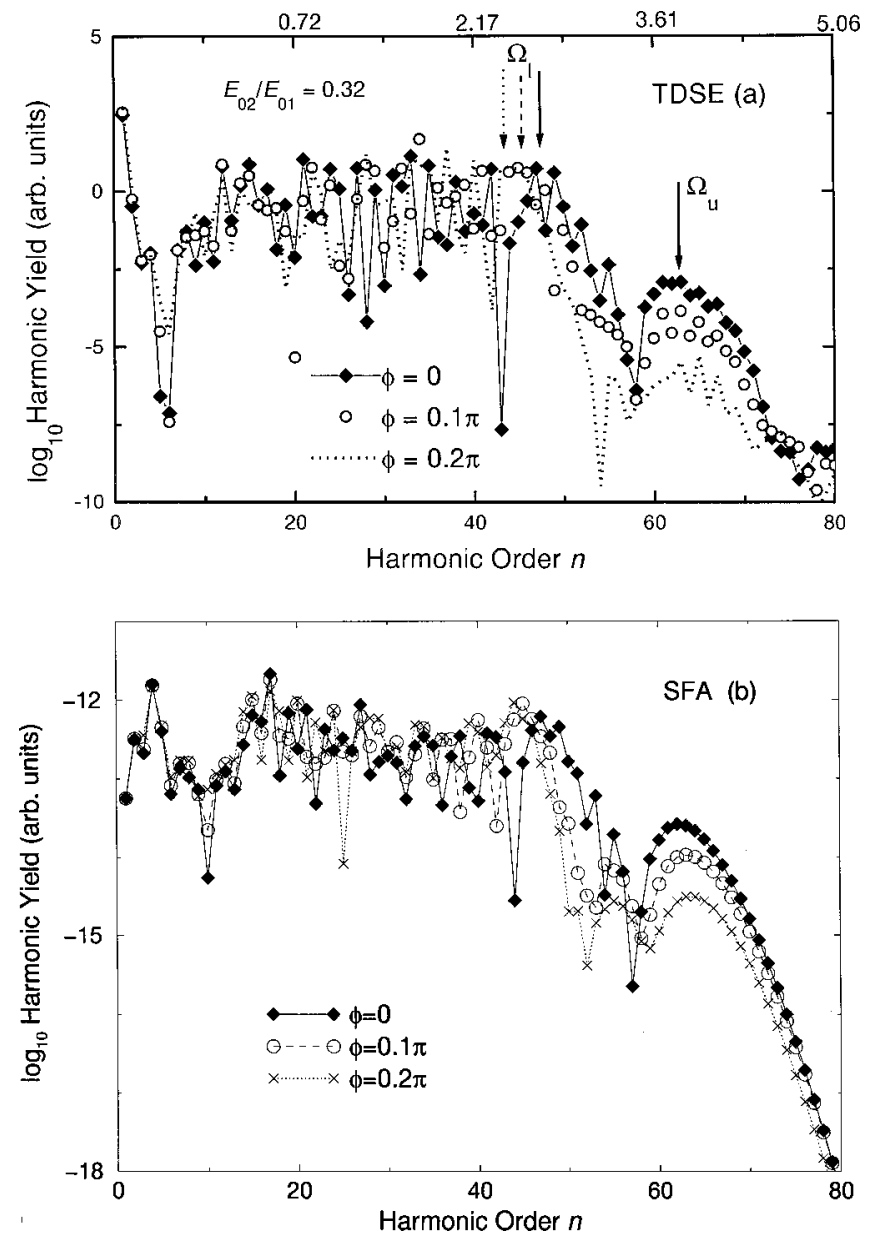

FIG. 3. Harmonic yields as functions of the harmonic order $n$ calculated using the TDSE [part (a)] and the strong-field approximation [part (b)], for the same bichromatic field as in the previous figures, with $E_{01}=0.1$ a.u. and relative phase $0 \leqslant \phi \leqslant 0.2 \pi$. The numbers on the upper edge of part (a) give the corresponding kinetic energy $E_{\text {kin }}\left(t_{1}, t_{0}\right)$ in units of the ponderomotive energy $U_{p}$. The upper and lower cutoff branches are indicated by thick arrows in part (a). For the phases $\phi=0, \phi=0.1 \pi$, and $\phi=0.2 \pi$, the cutoff $\Omega_{l}$ is indicated by a solid, dashed, and dotted arrow, respectively.

In Fig. 4 we compare the SFA results to the spectra obtained using the saddle-point method. The SPM results are obtained taking only four relevant complex solutions for the times $t_{0}$ and $t_{1}$ in Eq. (7) (see Fig. 2). The results agree qualitatively with the TDSE and SFA results for $n>30$. This shows that the main contribution to the harmonic yield near both cutoff branches comes from the four complex trajectories which have been explicitly discussed in Sec. III A.

A more detailed investigation of the field strength $\left|E\left(t_{0}\right)\right|$ at the emission time explains part of the features observed in Figs. 3 and 4. Figure 5(a) displays this parameter for the lower and upper branches, for intensity ratios $I_{2 \omega-\omega}=0.1$ and 0.2 . We show only the behavior for $0 \leqslant \phi \leqslant \pi$, since the semiclassical trajectories obey a period of $\pi$ with respect to the phase, due to the symmetry $E(t, \phi+\pi)=-E(t$ $-T / 2, \phi)$. This property is discussed in detail in $[12,30]$. From the figure it is clear that for a wide phase interval, namely for $0 \leqslant \phi \leqslant 0.8 \pi$, the lower branch presents a consid-
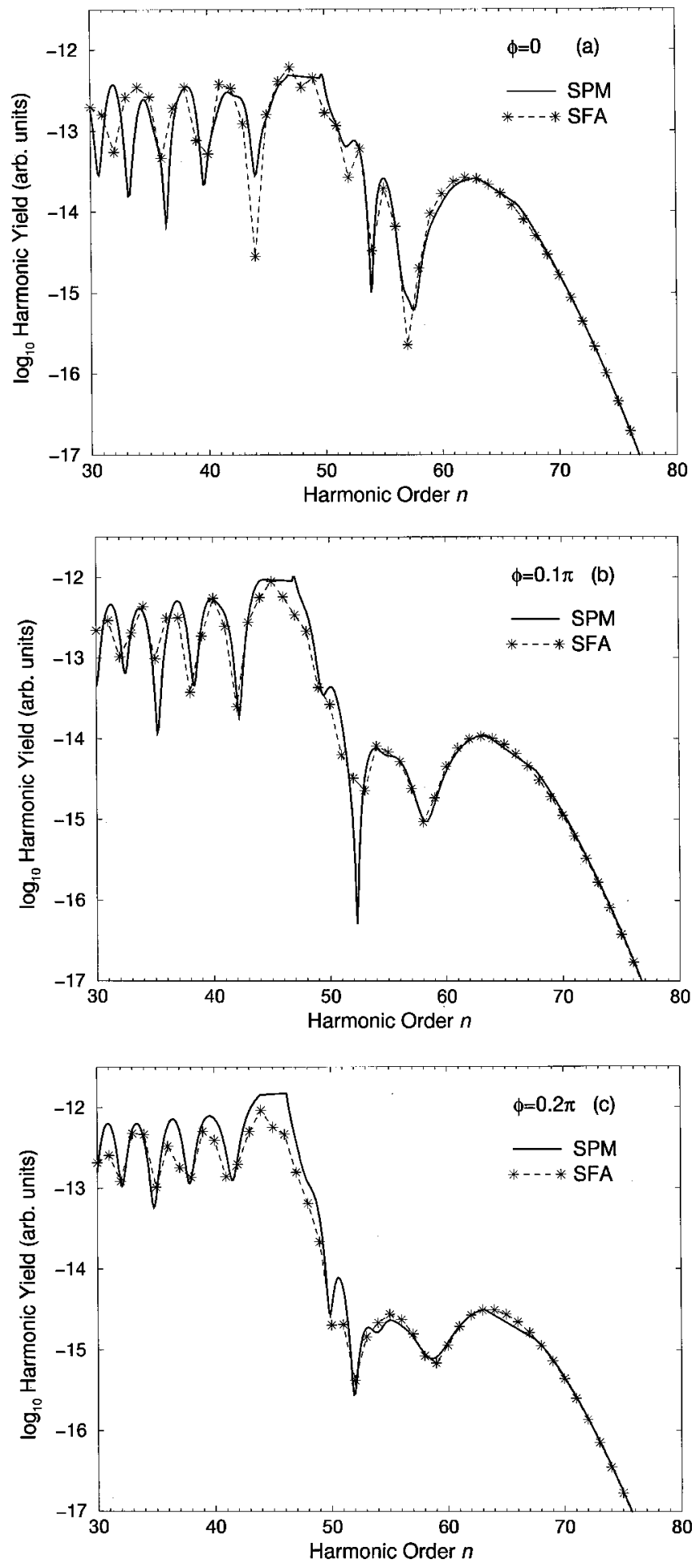

FIG. 4. Harmonic yields calculated with the saddle-point method (solid lines) compared to the strong-field approximation (stars connected by dashed lines) for the same bichromatic field and ionization potential as in Fig. 3, for (a) $\phi=0$, (b) $\phi=0.1 \pi$, and (c) $\phi=0.2 \pi$, as functions of the harmonic order $n$.

erably larger field at the emission time, such that its prominence in the spectrum is justified. This prominence decreases for the phase interval where both fields are comparable. Another important parameter is the excursion time of the electron in the continuum: the shorter the excursion time, the less 

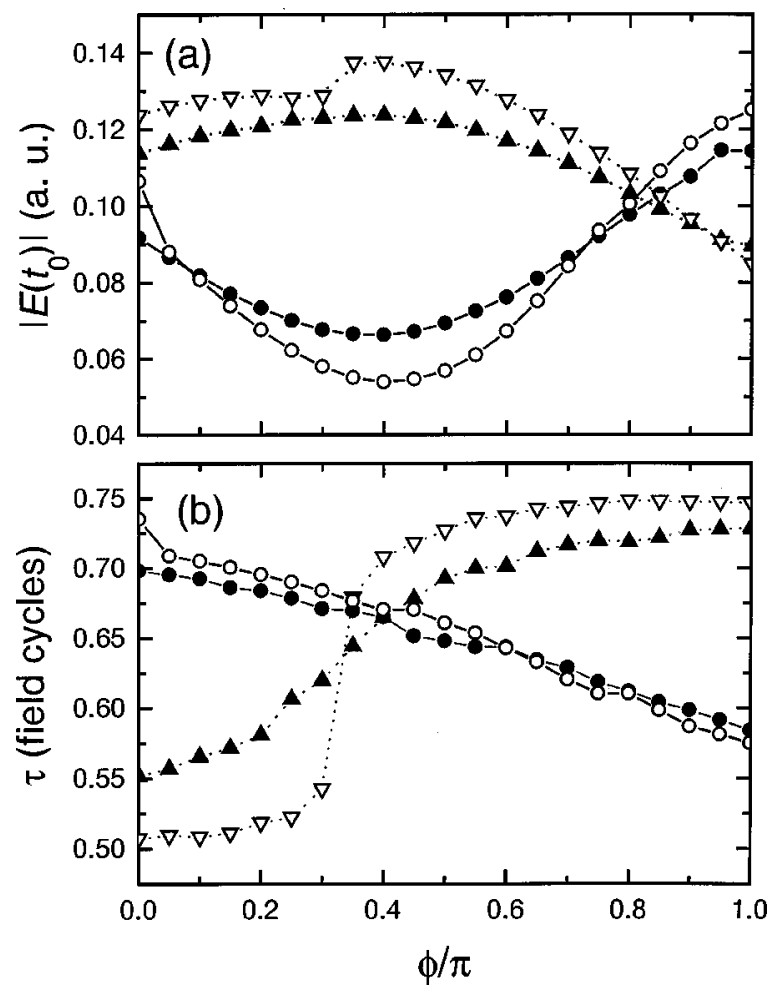

FIG. 5. Field strength $\left|E\left(t_{0}\right)\right|$ at the emission time [part (a)] and excursion times $\tau=t_{1}-t_{0}$ [part (b)], for the upper and lower cutoff branches, for intensity ratios $I_{2 \omega-\omega}=0.1$ (filled symbols), and $I_{2 \omega-\omega}=0.2$ (open symbols), as functions of the relative phase $\phi$. The triangles connected by dotted lines and the circles connected by solid lines correspond to the lower cutoff $\Omega_{l}$ and the upper cutoff $\Omega_{u}$, respectively.

important wave packet spreading. For both cutoff branches, these times are comparable and vary with the relative phase $\phi$. For phases smaller than $\phi \simeq 0.4 \pi$, the excursion time for the lower branch is slightly shorter than that of the upper branch. For the remaining phases, this pattern is reversed. This can be seen in Fig. 5(b), for the same intensity ratios as in the previous part of the figure. Thus, this does not appear to play a significant role in this case.

\section{Modulation and its phase shift}

For both lower and upper cutoff branches, the TDSE computation yields a modulation for the harmonic intensities as functions of the relative phase $\phi$, which is periodic in $\pi$. This $\pi$ periodicity is due to the symmetry in $E(t)$ mentioned in Sec. III B.

In order to study the offset phase shift, we investigate this modulation for consecutive harmonics near and slightly beyond the lower and upper cutoff branches. Figure 6(a) shows this variation for harmonics near $\Omega_{u}$ obtained from the quantum-mechanical computation, compared with the quasistatic rate for $E\left(t_{0 u}\right)$. The obvious coincidence between them indicates that for the upper branch the harmonic yield is determined by the quasistatic ionization rate, i.e., by the probability per unit time that the "first step" takes place. This is related to the fact that these harmonics are mainly deter-

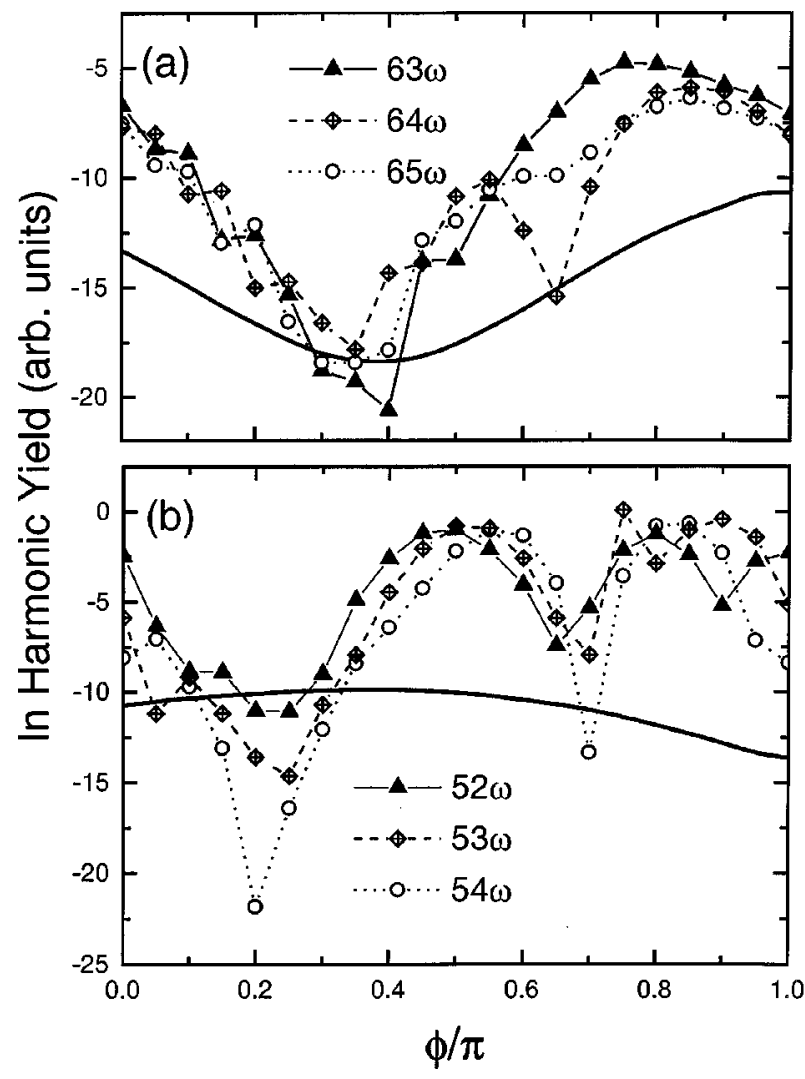

FIG. 6. Harmonic yield from the TDSE computation for neighboring cutoff harmonics compared to the quasistatic ionization rate, as functions of the relative phase $\phi$, for a driving field as in Fig. 3. Parts (a) and (b) refer to the cutoff branches $\Omega_{u}$ and $\Omega_{l}$, respectively. Part (a) displays the harmonics $\Omega=63 \omega, \Omega=64 \omega$, and $\Omega$ $=65 \omega$, whose energies are slightly larger than $\left|\varepsilon_{0}\right|+3.8 U_{p}$, while part (b) shows the harmonics $\Omega=52 \omega, \Omega=53 \omega$, and $\Omega=54 \omega$, with energies around $\left|\varepsilon_{0}\right|+3 U_{p}$ (slightly beyond the cutoff $\Omega_{l}$ ). The thick lines in the figure give the formula $-2^{5 / 2}\left|\varepsilon_{0}\right|^{3 / 2} / 3\left|E\left(t_{0}\right)\right|$, related to the quasistatic rate [see Eq. (4)], for $t_{0}=t_{0 u}[$ part (a) $]$ and $t_{0}=t_{0 l}[$ part (b) $]$.

mined by a single cutoff trajectory whose energy is almost independent of the relative phase $\phi$. Thus, other mechanisms that may influence the harmonic yield and produce a modulation, such as pronounced interference effects or significant variations in the harmonic intensities due to a shift in the cutoff energy, do not play a significant role.

On the other hand, for the lower cutoff branch the TDSE computation clearly shows a phase shift of the modulation for neighboring harmonics, which qualitatively corresponds to the feature reported in [21]. This phase shift is shown in Fig. 6(b), where the harmonic intensity is plotted as a function of the relative phase, for harmonics slightly beyond $\Omega_{l}$, namely at $\left|\varepsilon_{0}\right|+3 U_{p}$. It is strongly related to the variation of the cutoff energy with $\phi$. As a particular harmonic approaches or gets further in energy from $\Omega_{l}$, there is either an increase or a decrease in the harmonic yield. For a given phase, this intensity variation depends on the harmonic order, since different harmonics are unequally distant from the lower cutoff branch. Thus, neighboring harmonics present similar intensities for slightly different phases. For energies 
lower than or roughly at $\Omega_{l}$, there is also a pronounced interference structure superposed to the global behavior, which sometimes disguises this phase shift. This is not surprising since, in this energy region, $\Omega_{l}$ splits into two sets of trajectories for the returning electron which are temporally and energetically very close. For energies of the order of $\left|\varepsilon_{0}\right|+3 U_{p}$, these interference effects are practically absent.

Figure 6(b) also shows that the modulation observed does not follow the quasistatic ionization rate, given by the thick line in the figure. In fact, the modulation observed is a consequence of other physical mechanisms in addition to the tunneling process at the emission time, namely the energy variation of the lower cutoff branch and the interference between trajectories belonging to the lower and upper branches. As already discussed, the phase dependence of $\Omega_{l}$ is responsible for the phase shift of the modulation. Another example of its influence on the harmonic yield is a pronounced intensity drop seen in Fig. 6(b), which occurs for $\phi \simeq 0.25 \pi$. For this phase, the energy difference between the group of harmonics chosen and the cutoff $\Omega_{l}$ is most pronounced.

The offset phase shift is also present within the simpleman model, as described in Sec. II A for an electron ensemble with randomly distributed start times $0 \leqslant t_{0} \leqslant T$. This confirms the classical origin of this effect since, due to the strong phase dependence of $\Omega_{l}$, as $\phi$ is varied different amounts of electrons come from $\Omega_{l}$ and $\Omega_{u}$. Since electrons with slightly different $E_{\text {kin }}\left(t_{1}, t_{0}\right)$ are unequally distant in energy from the lower branch, the corresponding electron counts are also expected to be phase shifted with respect to each other. Figure 7 shows these counts as functions of the relative phase for different electron kinetic energies, which include contributions for one or both cutoff branches, depending on the phase in question. A more detailed behavior of $\Omega_{l}$ with the phase will be discussed below. In Fig. 7(a) we take into account the ionization rate given by Eq. (4), whereas in Fig. 7(b) we consider a constant ionization rate. The main difference between them is that, in Fig. 7(a), one of the two sets of peaks observed in Fig. 7(b) is strongly suppressed. Thus, $\left|E\left(t_{0}\right)\right|$ influences the modulation, but not its phase shift, only selecting the trajectories for which the field strengths at the electron start times are particularly strong.

The precise behavior of $\Omega_{l}$ and $\Omega_{u}$ with respect to the phase as calculated with the simple-man model is shown in Fig. 8 for several intensity ratios. Each point in the figure corresponds to an extremal kinetic energy for the returning electron. Figure 8(a) confirms that the cutoff energies $\Omega_{u}$ are very weakly influenced by the phase. The most important feature observed in the figure is the displacement of $\Omega_{u}$ towards higher energies for an increasing intensity of the highfrequency wave. This effect has been discussed in a previous paper [12]. On the other hand, for the cutoff $\Omega_{l}$ Fig. 8(b) shows a strong energy variation as $\phi$ is changed. An interesting feature is the energy minimum mentioned above at $0.25 \pi$ for intensity ratios of the order of or smaller than $I_{2 \omega} / I_{\omega}=0.2$. For stronger $2 \omega$ fields, the cutoff $\Omega_{l}$ splits into two, such that the interpretation of the results concerning the

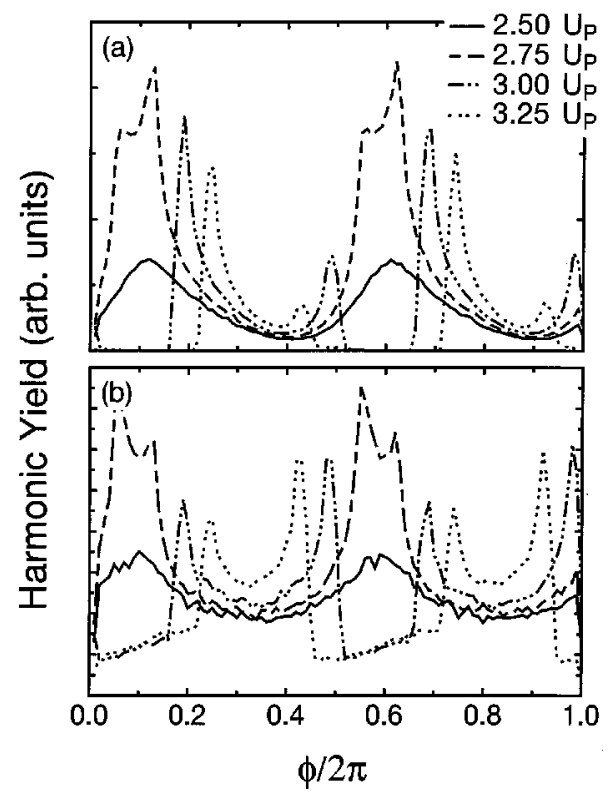

FIG. 7. Harmonic yields for various harmonic energies between $\Omega_{l}$ and $\Omega_{u}$ as functions of the relative phase $\phi$ calculated from the classical model. In part (a) the quasistatic ionization rate given by Eq. (4) and in part (b) a field-independent ionization rate was used. The calculation was performed with an ensemble of $5 \times 10^{7}$ electrons and randomly distributed start times $t_{0}$ and phases $\phi$. The kinetic energy at the return time $t_{1}$ was identified with the photon energy. The same field parameters as in Fig. 6 were used.

phase-dependent modulation becomes considerably more complicated.

The interference between the lower and upper cutoff branches plays only a secondary role in this modulation being, however, present for phases in the interval $0.5 \pi \leqslant \phi$ $\leqslant \pi$, for which $E\left(t_{0 l}\right)$ and $E\left(t_{0 u}\right)$ are comparable and the excursion time $\tau_{u}=t_{1 u}-t_{0 u}$ is shorter than that of the lower branch. Some information concerning these interferences can be obtained using the saddle-point method. In Fig. 9 we present the spectra resulting from isolated pairs of trajectories, compared to the results obtained taking into account all four relevant trajectories. Figure 9(a) displays these results for $\phi=0$, clearly showing that the lower cutoff branch is almost entirely determined by the trajectories $S_{1}$ and $S_{2}$. For $\phi=0.9 \pi$ [Fig. 9(b)], on the other hand, one clearly sees an interference pattern in the harmonics of the lower cutoff branch, originated by the trajectories $S_{3}$ and $S_{4}$.

\section{CONCLUSIONS}

We investigate high-order harmonic generation with bichromatic $\omega-2 \omega$ fields, giving a physical interpretation for the phase shift of the modulation observed experimentally for neighboring cutoff harmonics [21]. Using the threestep model and the fully numerical solution of the timedependent Schrödinger equation, we show that this feature is determined by the phase dependence of a set of semiclassical trajectories for the returning electron. With the introduction of the high-frequency driving wave, the cutoff for the monochromatic case splits into two branches, which exhibit differ- 


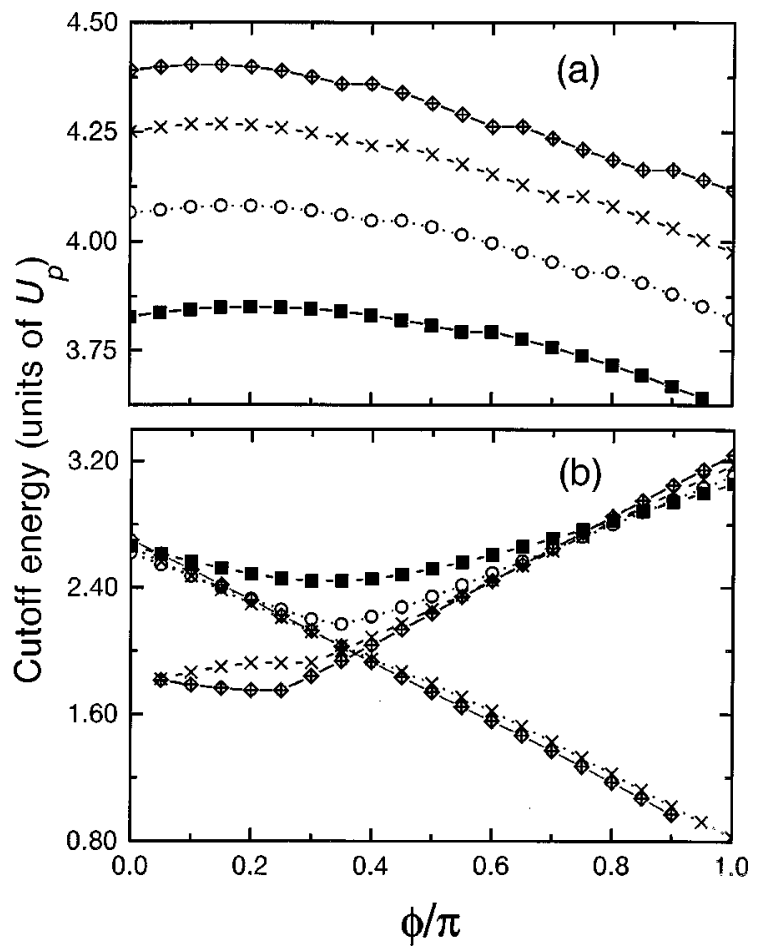

FIG. 8. Cutoff energy as a function of the phase $\phi$ for the upper [part (a)] and lower [part (b)] cutoff branches, given in terms of the ponderomotive energy $U_{p}$. The solid squares, open circles, crosses, and diamonds correspond to the intensity ratios $I_{2 \omega-\omega}=0.1$, $I_{2 \omega-\omega}=0.2, I_{2 \omega-\omega}=0.3$, and $I_{2 \omega-\omega}=0.4$, respectively. For intensity ratios larger than 0.2 , there is a splitting of the lower cutoff $\Omega_{l}$.

ent behavior with respect to the relative phase between the two driving fields. While the cutoff energy of the upper branch does not vary considerably with the phase, the cutoff energy of the lower branch is strongly sensitive to this parameter, giving rise to the phase shift of the modulation. Thus, using a two-color field, one can separate and identify a set of semiclassical trajectories already for a single atom, whereas for a monochromatic field, this is only possible using propagation effects of the harmonic radiation in the gaseous sample [14].

Using the saddle-point method, we are also able to make precise statements on how the interference between various trajectories influence the harmonic spectra, and reproduce the full quantum-mechanical results obtained with the TDSE for the harmonics close to the upper and lower cutoff branches with astonishing precision. We show that in the high plateau and cutoff regions, the harmonic intensities are well described by four interfering semiclassical trajectories for the returning electron. In particular, a single trajectory is responsible for the upper cutoff branch, whereas the lower branch is the result of the interference of three different trajectories.

Another noteworthy feature is the difference of orders of magnitude between the harmonic yields of the upper and lower branches. This is a direct consequence of a stronger field at the electron emission time for the cutoff $\Omega_{l}$ and therefore an interesting example of how groups of harmonics can be enhanced or suppressed by manipulating $\left|E\left(t_{0}\right)\right|$. Furthermore, the fact that the upper cutoff branch extends to
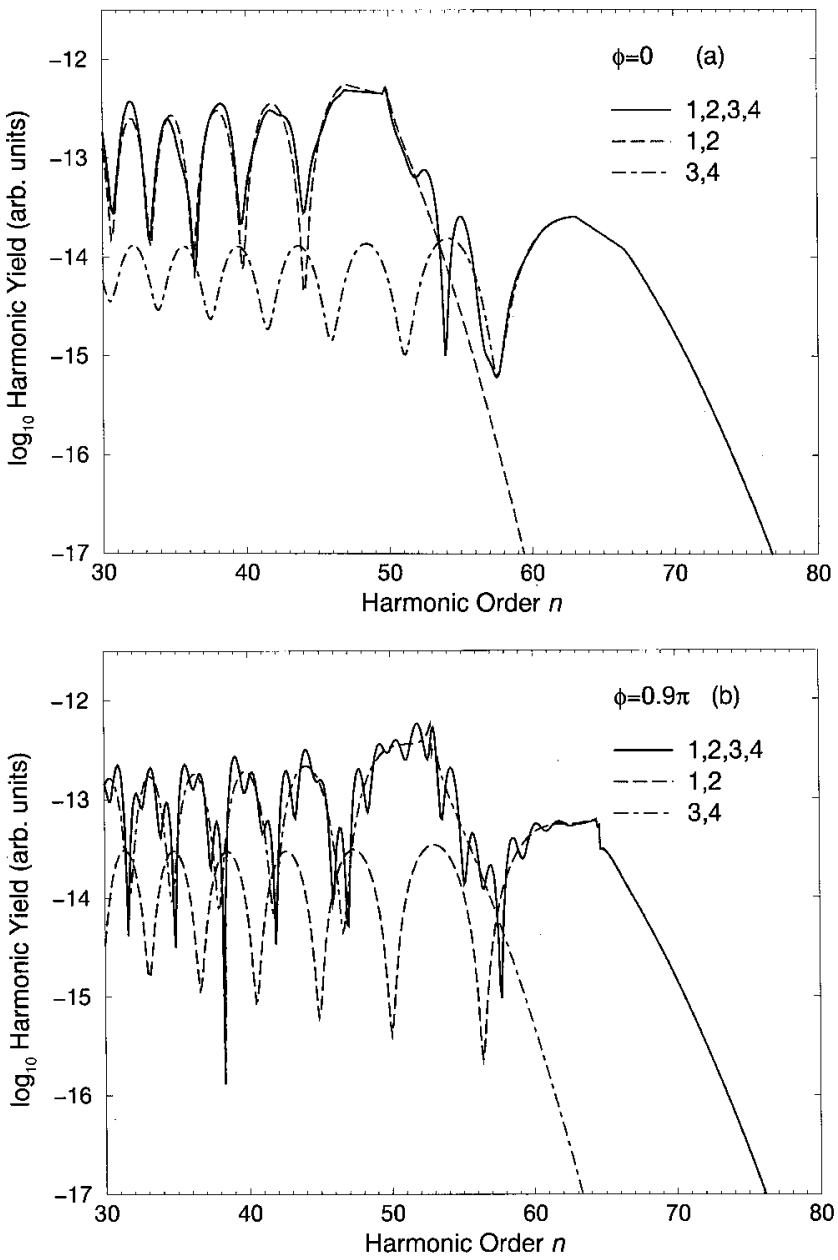

FIG. 9. Harmonic yields calculated using the SPM equations (7) and (8) for a hydrogenlike atomic potential with $\left|\varepsilon_{0}\right|=0.58$ a.u., and the same laser field parameters as in the previous figures, considering isolated pairs of semiclassical trajectories. Parts (a) and (b) correspond to the relative phases $\phi=0$ and $\phi=0.9 \pi$, respectively. The solid lines yield the contribution of the four relevant semiclassical trajectories, while the long-dashed and short-dashed lines correspond to the pairs $S_{1,2}$ and $S_{3,4}$, respectively.

energies higher than $\Omega_{\max }=\left|\varepsilon_{0}\right|+3.17 U_{p}$, but that the lower cutoff branch is more prominent in the spectra sheds some light into several apparently conflicting theoretical and experimental findings for high-order harmonic generation in bichromatic fields. In a large number of theoretical investigations, an extension of the plateau towards higher energies is observed, for $\omega-2 \omega[11,12,16]$ and $\omega-3 \omega$ [17] fields, whereas other theoretical and experimental studies yield a shorter $[18,19,21]$ plateau in comparison to the monochromatic cutoff energy $\Omega_{\max }$. Our results suggest that these studies refer either to the upper or to the lower cutoff branch, so that no contradiction exists. In particular, a double plateau was observed in [18] for a bichromatic driving field of frequencies $\omega$ and $2 \omega$, and the result found for the cutoff energy is in very good agreement with $\Omega_{l}$.

We also propose an explanation for the phase modulation observed in the harmonic yield for upper and lower cutoff branches. For the upper branch, this modulation is entirely 
determined by the quasistatic tunneling rate, whereas for the lower branch, it appears to be the combination of three main physical mechanisms, namely the field strength $\left|E\left(t_{0 l}\right)\right|$ at the emission time of the electron in the continuum, the energy variation of the cutoff energy $\Omega_{l}$ with the relative phase $\phi$, and the interference between the upper and lower cutoff branches, which plays a role when the fields at the emission time $\left|E\left(t_{0 l}\right)\right|$ and $\left|E\left(t_{0 u}\right)\right|$ are comparable for both branches.

Finally, we would like to comment on the cutoff measured in [21] for helium, whose energy was taken near the 15 th harmonic of the low-frequency field. This strong reduction in the cutoff energy was related to poor phase-matching conditions [31]. For this gas, however, the ionization poten- tial is roughly $\left|\varepsilon_{0}\right| \simeq 0.9$ a.u., which corresponds to approximately $\Omega=15 \omega$, with the frequency of the laser used being $\omega=0.057$ a.u. In this frequency region, the atomic internal structure strongly influences the harmonic spectra, such that the application or interpretation of the results in terms of the three-step model is questionable $[10,32]$.

\section{ACKNOWLEDGMENTS}

Discussions with W. Becker, R. Kopold, M. Dörr, E. Cormier, and M. Lewenstein are gratefully acknowledged. D. B. Milošević is supported by the Alexander von Humboldt Foundation.
[1] For a recent review, consult P. Salières, A. L'Huillier, Ph. Antoine, and M. Lewenstein, Adv. At., Mol., Opt. Phys. 41, 83 (1999).

[2] M. Yu. Kuchiev, Pis'ma Zh. Éksp. Teor. Fiz. 45, 319 (1987) [JETP Lett. 45, 404 (1987)]; K. C. Kulander, K. J. Schafer, and J. L. Krause, in Super-Intense Laser-Atom Physics, Vol. 316 of NATO Advanced Science Institute Series, Series B: Physics, edited by B. Piraux, A. L'Huillier, and K. Rzążewski (Plenum, New York, 1993); P. B. Corkum, Phys. Rev. Lett. 71, 1994 (1993).

[3] M. Lewenstein, Ph. Balcou, M. Yu. Ivanov, A. L'Huillier, and P. B. Corkum, Phys. Rev. A 49, 2117 (1994).

[4] W. Becker, S. Long, and J. K. McIver, Phys. Rev. A 41, 4112 (1990); 50, 1540 (1994).

[5] W. Becker, A. Lohr, M. Kleber, and M. Lewenstein, Phys. Rev. A 56, 645 (1997).

[6] See, e.g., M. Protopapas, C. H. Keitel, and P. L. Knight, Rep. Prog. Phys. 60, 389 (1997), and references therein.

[7] S. C. Rae, K. Burnett, and J. Cooper, Phys. Rev. A 50, 3438 (1994); R. Taïeb, A. Maquet, Ph. Antoine, and B. Piraux, in Super Intense Laser-Atom Physics IV, Proceedings of the NATO Advanced Workshop on SILAP IV, Moscow, Russia, 1995, edited by H. G. Muller and M. V. Fedorov (Kluwer, Dordrecht, 1996), p. 445.

[8] C. Figueira de Morisson Faria, M. Dörr, and W. Sandner, Phys. Rev. A 55, 3961 (1997).

[9] Ph. Antoine, B. Piraux, D. B. Milošević, and M. Gajda, Laser Phys. 7, 594 (1997).

[10] C. Figueira de Morisson Faria, M. Dörr, and W. Sandner, Phys. Rev. A 58, 2990 (1998).

[11] C. Figueira de Morisson Faria, M. Dörr, W. Becker, and W. Sandner, Phys. Rev. A 60, 1377 (1999).

[12] C. Figueira de Morisson Faria, W. Becker, M. Dörr, and W. Sandner, Laser Phys. 9, 388 (1999).

[13] A. de Bohan, Ph. Antoine, D. B. Milošević, and B. Piraux, Phys. Rev. Lett. 81, 1837 (1998); A. de Bohan, D. B. Milošević, G. L. Kamta, and B. Piraux, Laser Phys. 9, 175 (1999).

[14] M. Bellini, C. Lyngå, A. Tozzi, M. B. Gaarde, T. W. Hänsch, A. L'Huillier, and C.-G. Wahlström, Phys. Rev. Lett. 81, 297 (1998); M. B. Gaarde, F. Salin, E. Constant, Ph. Balcou, K. J.
Schafer, K. C. Kulander, and A. L'Huillier, Phys. Rev. A 59, 1367 (1999).

[15] M. Protopapas, P. L. Knight, and K. Burnett, Phys. Rev. A 49, 1945 (1994); T. Zuo, A. D. Bandrauk, M. Ivanov, and P. B. Corkum, ibid. 51, 3991 (1995); S. Long, W. Becker, and J. K. McIver, ibid. 52, 2262 (1995); D. M. Telnov, J. Wang, and S.-I. Chu, ibid. 52, 3988 (1995); W.-C. Liu and C. W. Clark, ibid. 53, 3582 (1996); M. Protopapas and P. L. Knight, J. Phys. B 28, 4459 (1995); M. B. Gaarde, A. L'Huillier, and M. Lewenstein, Phys. Rev. A 54, 4236 (1996); S. Varró and F. Ehlotzky, ibid. 56, 2439 (1997); A. D. Bandrauk, S. Chelkowski, H. Yu, and E. Constant, ibid. 56, R2537 (1997); $\mathrm{Ph}$. Antoine, D. B. Milošević, A. L'Huillier, M. B. Gaarde, P. Salières, and M. Lewenstein, ibid. 56, 4960 (1997); J. Z. Kamiński and F. Ehlotzky, J. Phys. B 30, 5729 (1997); X.-M. Tong and S.-I. Chu, Phys. Rev. A 58, R2656 (1998); F. Giammanco and P. Ceccherini, Laser Phys. 8, 593 (1998); Ph. V. Ignatovich, V. T. Platonenko, and V. V. Strelkov, J. Opt. Soc. Am. B 16, 435 (1999); S. Bivona, R. Burlon, and C. Leone, ibid. 16, 986 (1999); W. Becker, B. N. Chichkov, and B. Wellegehausen, Phys. Rev. A 60, 1721 (1999); V. Averbukh, O. E. Alon, and N. Moiseyev, ibid. 60, 2585 (1999).

[16] C. A. Ullrich, S. Erhard, and E. K. U. Gross, in Super Intense Laser Atom Physics IV (Ref. [7]), p. 477.

[17] M. Protopapas, A. Sanpera, P. L. Knight, and K. Burnett, Phys. Rev. A 52, R2527 (1995); A. Sanpera, J. B. Watson, M. Lewenstein, and K. Burnett, ibid. 54, 4320 (1996).

[18] D. B. Milošević and B. Piraux, Phys. Rev. A 54, 1522 (1996). We use the opportunity to mention a misprint in this reference: on the left-hand side in the first equation in Eq. (22) it should be $\vec{\pi}^{2}(t-\tau)$ and not $\vec{\pi}^{2}(t)$.

[19] M. D. Perry and J. K. Crane, Phys. Rev. A 48, R4051 (1993).

[20] H. Eichmann, S. Meyer, K. Riepl, C. Momma, and B. Wellegehausen, Phys. Rev. A 50, R2834 (1994); S. Watanabe, K. Kondo, Y. Nabekawa, A. Sagisaka, and Y. Kobayashi, Phys. Rev. Lett. 73, 2692 (1994); H. Eichmann, A. Egbert, S. Nolte, C. Momma, B. Wellegehausen, W. Becker, S. Long, and J. K. McIver, Phys. Rev. A 51, R3414 (1995); M. B. Gaarde, Ph. Antoine, A. Persson, B. Carré, A. L'Huillier, and C.-G. Wahlström, J. Phys. B 29, L163 (1996); K. Kondo, Y. Kobayashi, A. Sagisaka, Y. Nabekawa, and S. Watanabe, J. Opt. Soc. Am. B 13, 424 (1996). 
[21] U. Andiel, G. D. Tsakiris, E. Cormier, and K. Witte, Europhys. Lett. 47, 42 (1999).

[22] See, e.g., L. D. Landau and E. M. Lifshitz, Quantum Mechanics (Pergamon, Oxford, 1977).

[23] M. V. Ammosov, N. B. Delone, and V. P. Krainov, Zh. Éksp. Teor. Fiz. 91, 2008 (1986) [Sov. Phys. JETP 64, 1191 (1986)].

[24] G. G. Paulus, W. Becker, and H. Walther, Phys. Rev. A 52, 4043 (1995).

[25] M. Lewenstein, P. Salières, and A. L'Huillier, Phys. Rev. A 52, 4747 (1995).

[26] R. Kopold, D. B. Milošević, and W. Becker, Phys. Rev. Lett. 84, 17 (2000).

[27] G. van de Sand and J. M. Rost, Phys. Rev. Lett. 83, 524 (1999).

[28] In a one-dimensional computation, interference effects related to trajectories with long excursion times are overenhanced, since transversal wave-packet spreading is absent. In Eqs. (5) and (7), this neglect would give an exponent $-1 / 2$ instead of $-3 / 2$ in the $\tau$ prefactor.

[29] K. Burnett, V. C. Reed, J. Cooper, and P. L. Knight, Phys. Rev. A 45, 3347 (1992); J. L. Krause, K. Schafer, and K. Kulander, ibid. 45, 4998 (1992).

[30] K. J. Schafer and K. C. Kulander, Phys. Rev. A 45, 8026 (1992).

[31] See, e.g., A. L'Huillier, M. Lewenstein, P. Salières, P. Balcou,
M. Yu. Ivanov, J. Larsson, and C.-G. Wahlström, Phys. Rev. A 48, R3433 (1993); C.-G. Wahlström, J. Larsson, A. Persson, T. Starczewski, S. Svanberg, P. Salières, Ph. Balcou, and A. L'Huillier, ibid. 48, 4709 (1993); P. Saliéres, A. L'Huillier, and M. Lewenstein, Phys. Rev. Lett. 74, 3776 (1995); J. Peatross, M. V. Fedorov, and K. C. Kulander, J. Opt. Soc. Am. B 12, 863 (1995); Ph. Antoine, M. B. Gaarde, P. Salières, B. Carré, A. L'Huillier, and M. Lewenstein, in Proceedings of the VIIth International Conference on Multiphoton Processes, edited by P. Lambropoulos and H. Walther (IOP Publishing, Bristol, 1997), p. 142; J. Zhou, J. Peatross, M. M. Murnane, H. C. Kapteyn, and I. P. Christov, Phys. Rev. Lett. 76, 752 (1996); P. Salières, T. Ditmire, M. D. Perry, A. L'Huillier, and M. Lewenstein, J. Phys. B 29, 4771 (1996); Ph. Balcou, P. Saliéres, A. L'Huillier, and M. Lewenstein, Phys. Rev. A 55, 3204 (1997); I. P. Christov, M. M. Murnane, and H. C. Kapteyn, ibid. 57, R2285 (1998); M. B. Gaarde, Ph. Antoine, A. L'Huillier, K. J. Schafer, and K. C. Kulander, ibid. 57, 4553 (1998).

[32] K. S. Budil, P. Salières, A. L'Huillier, T. Ditmire, and M. D. Perry, Phys. Rev. A 48, R3437 (1993); N. H. Burnett, C. Kan, and P. B. Corkum, ibid. 51, R3418 (1995); K. Miyazaki and H. Takada, ibid. 52, 3007 (1995); M. Yu. Ivanov, T. Brabec, and N. Burnett, ibid. 54, 742 (1996). 